PROCESSO DE GESTÃO DE PROJETOS DE P\&D: UM ESTUDO DE CASO NA

\title{
CEMIG $^{1}$
}

P\&D Project Management: CEMIG Case Analysis

Adriana Carvalho de Menezes Dendena, Msc. PUC Minas dendena@uai.com.br

Dra Marta Araújo Tavares Ferreira Escola de Ciência da Informação, UFMG marta.tavarez@gmail.com

Dra Liliane de Oliveira Guimarães PPGA - PUC Minas lilianeog@pucminas.br

Dr. José Márcio de Castro PPGA - PUC Minas josemarcio@pucminas.br

Dra Ângela França Versiani PPGA - PUC Minas versiani@pucminas.br

Submissão: $15 / 08 / 2012$

Aprovação: 13/01/2013

\section{Resumo}

$\mathrm{O}$ presente artigo tem por objetivo discutir a gestão de projetos de Pesquisa e Desenvolvimento (P\&D) no programa CEMIG/ANEEL. A partir de entrevistas com os gerentes de projetos e de pesquisa documental, foram identificados os processos utilizados na gestão de seis projetos considerados de maior potencial para a transferência de resultados à indústria. A revisão da literatura apontou características de cinco gerações de modelos de gestão de $\mathrm{P} \& \mathrm{D}$, fundidos no modelo teórico concebido para a análise dos dados. A análise do modelo de gerenciamento adotado pelo programa CEMIG/ANEEL, mesmo não permitindo a sua classificação em uma determinada geração, sugere, no entanto, que o modelo privilegia características da segunda geração de gestão de P\&D.

Palavras-chave: gestão de projetos, modelos de gestão de P\&D, programa CEMIG/ANEEL

\footnotetext{
${ }^{1}$ os autores agradecem a CEMIG/ANEEL pelo apoio financeiro e logístico para a realização da pesquisa.
} 


\begin{abstract}
This article aims to discuss the $\mathrm{P} \& \mathrm{D}$ project management in the CEMIG/ANEEL program. Based on interviews with managers as well as secondary data, we mapped the processes of six management projects whose results could be potentially transferred to firms. The literature review pointed out characteristics of five generations of $P \& D$ management models which, by its turn, were used for building our theoretical framework. The analysis of the program did not allow us to classify it into a particular generation, although it shares some characteristics of the second generation model.
\end{abstract}

Key-words: project management, P\&D management models, CEMIG/ANEEL program 


\section{Introdução}

Como parte do esforço brasileiro para fomentar as atividades de P\&D no país, a Lei n 9.991, de 24 de julho de 2000 (BRASIL, 2000), estabeleceu percentuais mínimos a serem investidos em programas de pesquisa e desenvolvimento (P\&D) pelas empresas concessionárias, permissionárias e autorizadas do setor elétrico brasileiro. Apenas as empresas que geram energia exclusivamente a partir de pequenas centrais hidrelétricas, de biomassa, usinas eólicas ou solares ficaram isentas de cumprir essa lei (BRASIL, 2000), que estipula que cada empresa do setor elétrico submeta, anualmente, à aprovação da Agência Nacional de Energia Elétrica (ANEEL), um programa de P\&D contendo metas físicas e financeiras.

Desta forma, verifica-se que significativos volumes de recursos estão sendo investidos em pesquisa e desenvolvimento na área de energia por concessionárias de eletricidade, fundos setoriais de energia, petróleo e gás, além dos tradicionais recursos do sistema público de fomento representado pelo Conselho Nacional de Desenvolvimento Científico e Tecnológico (CNPq), pela Financiadora de Estudos e Projetos (FINEP) e pelas Fundações de Amparo à Pesquisa estaduais (FAP’s) (JANNUZZI, 2003).

Com a Lei 9.991, buscou-se reforçar a cultura de P\&D nas empresas do setor elétrico brasileiro e sensibilizá-las para a gestão da inovação. Esta preocupação responde à percepção atual de que o gerenciamento do processo de inovação no âmbito de empresas é um importante instrumento de geração de riqueza e desenvolvimento socioeconômico (RENAULT et al., 2007).

O presente artigo tem por objetivo discutir a gestão dos projetos de $\mathrm{P} \& \mathrm{D}$ no âmbito do programa CEMIG/ANEEL, basicamente, os projetos aprovados e iniciados entre anos 2000 e 2005 e finalizados até fevereiro de 2008 , ou seja, aqueles tinham maior potencial de transferência tecnológica para a indústria. Os projetos concluídos nesse período, em sua grande maioria, geraram algum tipo de protótipo, processo ou software, mas que, raramente, foram transferidos para a indústria ou foram utilizados pela própria CEMIG.

A análise do modelo de gestão desses projetos de P\&D foi considerada fundamental para que se determinassem os elementos que dificultaram a transferência dos resultados para o setor industrial e a geração de inovações. Com este objetivo, foram identificados os processos e mecanismos utilizados na gestão de projetos de $\mathrm{P} \& \mathrm{D}$ do programa CEMIG/ANEEL iniciados entre 2000 e 2005 e finalizados até fevereiro de 2008. 


\section{Evolução da gestão de $P \& D$ e de sua relação com a inovação}

A gestão dos projetos de $\mathrm{P} \& \mathrm{D}$ tem sido objeto de preocupação de gestores organizacionais em virtude da necessidade de compatibilizar os objetivos estratégicos, seus recursos e assegurar a ênfase no desenvolvimento de projetos prioritários (ROUSSEL et al., 1991; REED et al. 1996; COOPER et al., 1996, 1999; DRUCKER, 1998; SAWHNEY; PRANDELLI, 2000).

Para se identificar o modelo de gestão de P\&D que está sendo utilizado é necessário conhecer as principais características dos modelos de gestão de $\mathrm{P} \& \mathrm{D}$ existentes, que são discutidas por diversos autores. Maximiniano (1997), por exemplo, argumenta que devem ser considerados dois importantes aspectos na gestão de P\&D: a gestão do projeto em si e gestão de um projeto no contexto organizacional. O primeiro aspecto inclui um projeto integrado de gestão de recursos humanos, materiais, financeiros e atividades a serem realizadas em prazos específicos. O segundo aspecto é o de administrar o projeto como atividade coletiva na organização, o que implica capacidade de planejar e organizar recursos com vistas às metas propostas.

Vários aspectos na avaliação em gestão de projetos podem afetar os resultados da $\mathrm{P} \& \mathrm{D}$, entre eles, a estrutura DE coordenação, a definição de resultados a serem obtidos, a política de recursos humanos, a gestão da informação para a disseminação e reutilização em outros projetos, o perfil do gestor de projetos, a gestão da qualidade e, por fim, o investimento da organização em capacitação de pesquisadores para a atuação em gestão de projetos (LIMA, 1999).

A gestão de pesquisa para a inovação exige que o gerente lide com múltiplos objetivos e variáveis operacionais da organização que influenciam no planejamento, nas decisões e na avaliação. Para vários autores, a gestão de $\mathrm{P} \& \mathrm{D}$ é um processo permanente que inclui a geração, difusão, transferência de conhecimento entre diferentes atores, tais como, indivíduos, grupos e organizações. Como a P\&D não é realizada de maneira isolada, ou seja, é dependente de fontes externas, as competências requeridas para o gestor envolvem não só a gestão da pesquisa, mas, também, sua capacidade de estabelecer conexões, redes e relacionamentos externos (METCALF; BODEN, 1991; COOPER; EDGET; KLEINSCHMIDT, 1997; DANNEELS; KLEINSCHMIDT， 2001; MCCORMACK et al., 2001; NELLORE; BALACHANDRA, 2001; SETHI; NICHOLSON, 2001). Além disso, como a gestão de P\&D é interativa, faz-se essencial que o gestor desenvolva uma visão integrada de todas as etapas que compõem um projeto, o 
que inclui a utilização de instrumentos institucionais, recursos humanos e materiais (CARVALHO, 2002).

A literatura que discute os componentes da gestão de P\&D define modelos de gestão de P\&D classificados como "gerações de gestão de P\&D”. Rothwell (1991, 1994) apresenta um modelo de cinco gerações de gestão de $\mathrm{P} \& \mathrm{D}$, a saber: i) technology-push; ii) market pull; iii) integração de P\&D com a estratégia de negócio; iv) inovação contínua; e, v) Modelo multi-institucional de integração para a inovação. O modelo technology-push configura-se como a primeira delas tendo predominado nos anos cinquenta e meados dos anos sessenta. Lichtenthaler (2003) esclarece que, nesta primeira geração de gestão de $\mathrm{P} \& \mathrm{D}$, não há uma relação direta entre a estratégia da organização e a atividade de $\mathrm{P} \& \mathrm{D}$, que responde diretamente aos desafios abertos pelo avanço da tecnologia, daí ser a expressão technology-push.

Para Roussel et al. (1992), Liyanage et al. (1999) e Lichtenthaler (2003) o modelo de primeira geração de gestão de $\mathrm{P} \& \mathrm{D}$ é caracterizado pela ausência de planejamento estratégico para a gestão da tecnologia e da Pesquisa e Desenvolvimento. A P\&D é tratada como custo e a administração superior tem participação discreta na definição do programa de pesquisa, sendo este uma responsabilidade exclusiva da gerência de P\&D. Nestes termos, a definição do portfólio de projetos de $\mathrm{P} \& \mathrm{D}$ é, muitas vezes, baseada na percepção dos pesquisadores sobre a importância da geração do novo conhecimento, não existindo um planejamento, especificando objetivos, recursos, prazos e dispêndios e a gerência de P\&D pouco se comunica com os demais setores da empresa (ROUSSEL et al., 1991, 1992).

A primeira geração de gestão empresarial de $P \& D$ privilegia a criação de conhecimento científico. A liberdade do pesquisador é considerada muito importante e poucas técnicas formais são adotadas para a seleção e avaliação dos projetos de P\&D. Para (LIYANAGE et al., 1999). Trata-se de um modelo que se mostra bastante atraente para os pesquisadores, pois há pouca pressão por resultados (BURNS; STALKER, 1961; IRVINE; MARTIN, 1984).

O modelo need-pull, predominante na metade dos anos sessenta e início dos anos setenta, é o protótipo da segunda geração de P\&D (ROTHWELL, 1991) e caracteriza-se pela seleção de projetos de P\&D baseada em propósitos e objetivos definidos. Esse modelo responde ao conceito de market pull, reiterando a importância em se obter conhecimento que resulte em resultados econômicos (MOWERY; ROSENBERG, 1979). Nesses termos, 
os projetos de $\mathrm{P} \& \mathrm{D}$ devem gerar resultados tangíveis em um período específico, o que leva à maior necessidade de comunicação entre os gerentes de pesquisa e as outras áreas da empresa (ROUSSEL et al., 1991).

Nessa segunda geração, o controle financeiro, contábil e a avaliação de projetos são introduzidos no gerenciamento de P\&D. Liyanage et al. (1999) e Jung, Ribeiro e Caten (2008) listam como principais características do modelo de gestão de P\&D de segunda geração: i) a estratégia orientada para o mercado; ii) projetos objetivos; iii) melhores métodos de avaliação de projetos; e, iv) exigência de qualidade de projeto.

Mowery e Rosenberg (1979), Irvine e Martin (1984), Liyanage et al. (1999) e Reis (2008) argumentam que esse modelo leva em consideração, no investimento de P\&D, a demanda do mercado mais do que a disponibilidade de conhecimento científicotecnológico. O objetivo principal desse modelo gerencial é a inovação tecnológica, conseguida a partir da pressão do mercado, o que gera uma pesquisa aplicada com resultados testados experimentalmente até que a inovação seja realizada.

A terceira geração, desenvolvida ainda no início dos anos setenta e meados dos anos oitenta, enfatizou tanto a relação entre $P \& D$ e a área mercadológica quanto a combinação entre technology-push (estímulo da tecnologia) e marketpull (estímulo do mercado). O modelo de terceira geração de gestão de $\mathrm{P} \& \mathrm{D}$ se caracteriza pela perspectiva de que a $\mathrm{P} \& \mathrm{D}$ pode ser melhor gerenciada quando está relacionada às estratégias empresariais. Nesses termos, a comunicação eficiente entre pesquisadores e gerentes é considerada necessária para integrar estratégia e funções operacionais da organização.

A definição dos projetos de P\&D leva em consideração a maturidade tecnológica e os impactos na competitividade; a alocação de recursos é baseada no balanço entre prioridade e risco; a análise da consistência das metas está relacionada com o negócio e os objetivos tecnológicos; o estabelecimento de prioridades ocorre de acordo com a relação custo/benefício e a avaliação da contribuição da P\&D para os objetivos organizacionais.

O processo de avaliação dos resultados torna-se regular e conforme as necessidades de desenvolvimento interno e externo (ROUSSEL et al., 1992; JUNG, RIBEIRO, CATEN, 2008; LIYANAGE et al., 1999). Para Burgess e Smitham (1995), esse modelo de gestão de $\mathrm{P} \& \mathrm{D}$ de terceira geração é adequado para empresas maiores que se preocupam com maior coordenação e controle do portfólio de P\&D.

Um novo modelo chamado de gestão de P\&D de quarta geração diferencia-se do modelo anterior pela sua abordagem da inovação. Para os autores, a inovação contínua ou 
descontínua é mandatária para se lidar com aceleradas mudanças que então invadem o mercado (MILLER; MORRIS apud LIYANAGE et al., 1999).

Os autores propõem basicamente um modelo de inovação baseada na quebra de paradigmas e na fusão de novas tecnologias. Para tanto, argumentam que novos conhecimentos e uma nova arquitetura organizacional são necessários. Além disso, o modelo de quarta geração de gestão de $\mathrm{P} \& \mathrm{D}$ caracteriza-se pela integração do desenvolvimento interno da inovação com a colaboração externa (MILLER; MORRIS apud LIYANAGE et al., 1999). Salter e Martin (2001) complementam essas características da quarta geração de gestão de $\mathrm{P} \& \mathrm{D}$, incluindo aspectos como foco e interação com o cliente, gestão da inovação e do conhecimento e equilíbrio entre objetivos de curto, médio e longo prazo.

Liyanage et al. (1999) vão além de Miller e Morris (apud LIYANAGE et al. 1999) e Salter e Martin (2001) apontando que a quarta geração de gestão de P\&D está centrada em três requisitos principais: i) criatividade; ii) uso e fortalecimento de redes de relacionamento; e, iii) exploração do conhecimento.

A criatividade resulta na manifestação de novas idéias em inovações radicais, evita a inércia da pesquisa e previne para que o fluxo de conhecimento não seja interrompido, ou seja, incentiva a busca constante de conhecimentos. E, se a gestão do conhecimento interno é considerada a condição mais importante para a competitividade do negócio e melhoria da tecnologia (ROOME, 1994) as relações externas, por meio de redes, dinamizam a criação de conhecimento e determinam o ritmo e a direção da inovação.

O modelo de quarta geração prevê a participação de vários grupos de interesse (stakeholders) na pesquisa, produção, inovação e comercialização de tecnologia (ARNOLD; GUY, 1986; HASKLISCH et al., 1986; DUMAINE, 1989; ROTHWEL, 1991; DODGSON, 1993). Entretanto, para Coutinho (2004), o ideal é que se inclua nesse esforço não somente fornecedores e clientes e, sim, toda a cadeia produtiva, ou seja, devem participar da busca por inovações os fornecedores dos fornecedores e os clientes dos clientes. Cabe às empresas selecionar a(s) cadeia(s) de maior valor estratégico para seus negócios e, nesta(s), identificar os principais agentes de inovação.

As principais características do modelo de quarta geração de gestão de P\&D podem ser sintetizadas da seguinte forma: i) gestão estratégica do conhecimento interno da organização conjugado com fontes externas; ii) existência de sistema ativo de informações e de comunicação; iii) existência de redes de relações; e, iv) interação para a gestão do 
fluxo de informações e integração entre pesquisa, produção e inovação. A gestão do conhecimento por meio de redes é parte essencial da quarta geração de gestão de P\&D (LIYANAGE et al., 1999). O modelo de quarta geração de gestão de P\&D também enfatiza a necessidade de aplicação e transferência de conhecimentos para além das fronteiras institucionais, preconizando que a transição de um ambiente de pesquisa para um ambiente de produção requer um processo de aprendizagem organizacional específico.

No modelo de Liyanage et al. (1999) para realização dos projetos, os principais insumos são: o conhecimento das equipes de pesquisa nas várias disciplinas envolvidas na solução do problema que o projeto busca alcançar; a criatividade dessas equipes e dos técnicos envolvidos nos projetos, seja na formulação de soluções para os problemas, seja na condução metodológica; recursos materiais e financeiros específicos para os projetos; conhecimento sobre as demandas tecnológicas do mercado.

Por fim, a Gestão de P\&D de quinta geração, com início nos anos de 1990 (ROTHWELL, 1991, 1992), é compreendida como um modelo multi-institucional de integração entre inovação e estratégia empresarial, incluindo a participação de clientes e fornecedores primários e fortes encadeamentos horizontais.

O modelo de gestão de $\mathrm{P} \& \mathrm{D}$ de quinta geração possui elementos estratégicos que dizem respeito à relação com o tempo, ou seja, quanto mais rápido um produto for lançado no mercado, maior será considerada a eficiência do seu desenvolvimento. Ser o primeiro com um novo produto no mercado que oferece benefícios econômicos para os consumidores representa vantagem competitiva, favorecendo e aumentando a satisfação dos clientes (REINER, 1989). Rudolph (1989) e Crawford (1992), por outro lado, esclarecem que empresas que não acompanham a velocidade do mercado sofrem diversas penalidades.

As principais características dos cinco modelos de gestão de P\&D são descritas resumidamente no Quadro 1. 
QUADRO 1

\section{Características das gerações de gestão de P\&D}

\begin{tabular}{|c|c|c|c|}
\hline GERAÇÕES & CARACTERÍSTICAS & PERÍODO & AUTORES \\
\hline $1^{a}$ & $\begin{array}{l}\text { Gerência de P\&D: área isolada, autonomia na } \\
\text { seleção e condução das pesquisas. } \\
\text { Estratégia science push: estímulo à ciência, } \\
\text { privilégio para pesquisas e criação de conhecimento } \\
\text { científico (pesquisadores individuais). Gerência } \\
\text { Geral: pouca participação com P\&D. Ausência de } \\
\text { planejamento estratégico. }\end{array}$ & $\begin{array}{l}1950 \text { até } \\
\text { metade de } \\
1960\end{array}$ & $\begin{array}{l}\text { Burns; Stalker (1961); } \\
\text { Irvine; Martin (1984); } \\
\text { Roussel } \\
\text { et al. (1991, 1992); } \\
\text { Rothwell } \\
\text { (1991,1994); } \\
\text { Lyianage et al. (1999); } \\
\text { Lichtenthaler (2003). }\end{array}$ \\
\hline $2^{a}$ & $\begin{array}{l}\text { Qualidade dos projetos. Início de um quadro } \\
\text { estratégico de P\&D. Estratégia market push: } \\
\text { orientada para o mercado com resultados } \\
\text { econômicos. Projetos objetivos e melhores métodos } \\
\text { de avaliação dos projetos. Melhor comunicação } \\
\text { entre gerentes de pesquisas e gerentes gerais. } \\
\text { Seleção dos projetos com propósitos e objetivos } \\
\text { definidos com período específico. }\end{array}$ & $\begin{array}{l}\text { Metade de } \\
1960 \text { até } \\
\text { início de } \\
1970\end{array}$ & $\begin{array}{l}\text { Rosenberg (1979); } \\
\text { Irvine, Martin (1984); } \\
\text { Rothwell } \\
\text { (1991); Roussel et al. } \\
\text { (1991); Lyianage et } \\
\text { al. (1999); Mowery, } \\
\text { Reis (2008); Jung, } \\
\text { Ribeiro, Caten } \\
\text { (2008). }\end{array}$ \\
\hline $3^{a}$ & $\begin{array}{l}\text { Melhor gerenciamento de P\&D; comunicação } \\
\text { eficiente; integração da estratégia empresarial com } \\
\text { as funções operacionais da organização; avaliação } \\
\text { dos resultados de P\&D; maior coordenação e } \\
\text { controle de portfólios de P\&D. }\end{array}$ & $\begin{array}{l}\text { Início de } \\
1970 \text { até } \\
\text { metade } \\
\text { de } 1980\end{array}$ & $\begin{array}{l}\text { Roussel et al. (1991, } \\
\text { 1992); Burgess, } \\
\text { Smitham } \\
\text { (1995); Lyianage et al. } \\
\text { (1999); Jung, Ribeiro, } \\
\text { Caten (2008). }\end{array}$ \\
\hline $4^{a}$ & $\begin{array}{l}\text { Inovação contínua; integração funcional; interação } \\
\text { com o cliente; criatividade; fortalecimento de redes } \\
\text { de relacionamento; exploração do conhecimento; } \\
\text { ampliação e transferência de conhecimentos. }\end{array}$ & $\begin{array}{l}\text { Metade de } \\
1980 \text { até } \\
\text { início de } \\
1990\end{array}$ & $\begin{array}{l}\text { Dumanine (1989); } \\
\text { Hagedoorn (1990); } \\
\text { Bessant (1991); } \\
\text { Dodgson (1993); } \\
\text { Miller, Morris, apud } \\
\text { Lyianage et al. (1999); } \\
\text { Coutinho (2004). }\end{array}$ \\
\hline $5^{\mathrm{a}}$ & $\begin{array}{l}\text { Modelo multi-institucional de integração para a } \\
\text { inovação; participação de clientes; } \\
\text { integração estratégica; qualidade; adaptação à } \\
\text { velocidade das mudanças impactando as inovações. }\end{array}$ & $\begin{array}{l}\text { Início de } \\
1990\end{array}$ & $\begin{array}{l}\text { Reiner (1989); Rudolph } \\
\text { (1989); Rothwell (1991, } \\
\text { 1992); } \\
\text {.Crawford (1992). }\end{array}$ \\
\hline
\end{tabular}

Quadro 1: Características das gerações de gestão de P\&D

Fonte: DENDENA (2011).

Em resumo, a gestão de $P \& D$ de quinta geração prioriza fatores relacionados com a qualidade em relação ao preço. Também enfatiza a flexibilidade e a responsabilidade corporativa; estratégia empresarial direcionada ao atendimento dos clientes; estratégia de integração com fornecedores; estratégia de colaboração tecnológica horizontal; política de qualidade total e informatização. 


\section{Procedimentos metodológicos}

A pesquisa realizada na CEMIG foi de natureza qualitativa, o que, segundo Richardson (1999) [...] pode ser caracterizada como a tentativa de uma compreensão detalhada dos significados e características situacionais apresentadas pelos entrevistados, em lugar da produção de medidas quantitativas de características ou comportamentos (RICHARDSON, 1999, p. 90). O método utilizado foi o estudo de caso que se caracteriza como “(...) um tipo de pesquisa cujo objeto é uma unidade que se analisa profundamente. Visa ao exame detalhado de um ambiente, de uma dada unidade social" (Godoy, 1995, p. 25).

Conforme já mencionado, o objetivo deste trabalho foi o de compreender o programa de $\mathrm{P} \& \mathrm{D}$ desenvolvido pela concessionária de energia elétrica CEMIG, após a instituição da obrigatoriedade de destinação de recursos à pesquisa, bem como de analisar a gestão dos projetos de $\mathrm{P} \& \mathrm{D}$, identificando os mecanismos de coordenação e controle.

Para atingir este objetivo, inicialmente foi realizada uma primeira reunião com os gerentes da Superintendência de Tecnologia e Alternativas Energéticas da CEMIG para mapeamento da população do estudo. Ficou estabelecido que os projetos iniciados em 2005 e finalizados até fevereiro de 2008 seriam considerados e a justificativa era de que os seus resultados pudessem ser conhecidos. Sem seguida, foram obtidas algumas informações sobre as características técnicas desses projetos, como produtos, publicações, capacitações realizadas, entre outros.

Para a análise da gestão dos projetos de P\&D, foi sugerido pelos gerentes da CEMIG que fosse realizado um estudo aprofundado de seis projetos de $\mathrm{P} \& \mathrm{D}$, a partir de um conjunto de 22 projetos cujos resultados, em princípio, apresentavam maior interesse para industrialização. Os projetos de $\mathrm{P} \& \mathrm{D}$ escolhidos para análise em profundidade foram: P\&D 003, P\&D 006, P\&D 023, P\&D 051, P\&D 084 e P\&D 110.

Em seguida foram realizadas entrevistas sobre a gestão dos projetos de P\&D com os gerentes dos projetos selecionados. Em complemento, a análise documental foi outro procedimento de coleta de dados utilizado, recorrendo-se, então, a documentos legislativos e regulatórios do setor elétrico, ao manual da ANEEL para o desenvolvimento de pesquisas, além de documentos relacionados aos próprios projetos de $\mathrm{P} \& \mathrm{D}$ selecionados para o estudo.

Para a identificação dos resultados, foram utilizados os resumos dos projetos de P\&D selecionados, disponibilizados ao público na página eletrônica da agência reguladora. 
Cada resumo contém o título do projeto, seus objetivos, linha de pesquisa, entidade executora e custo total.

As entrevistas foram realizadas a partir de um roteiro semi-estruturado. Novas questões surgiram e foram incorporadas ao longo do trabalho. As entrevistas foram realizadas com os seis gerentes dos projetos de P\&D selecionados e geraram informações tais como: origem do projeto de pesquisa em questão; seleção dos parceiros; interação entre os executores do projeto e o gerente CEMIG durante o desenvolvimento do projeto; acompanhamento da evolução do mercado e da tecnologia ao longo do projeto; principais resultados e seu interesse potencial para a indústria e para as operações da CEMIG; solicitação ou não de proteção da propriedade intelectual; absorção dos resultados pela CEMIG, ou pela indústria; principais dificuldades encontradas na transferência dos resultados a indústria; ganhos obtidos pela CEMIG; ganhos para o setor acadêmico e para o empresarial; e, finalmente, sugestões de modificações no gerenciamento dos projetos CEMIG/ANEEL.

\subsection{Modelo conceitual e estratégia de análise dos dados}

A partir da revisão de literatura, elaborou-se um modelo para análise dos dados, conforme o Figura 2.

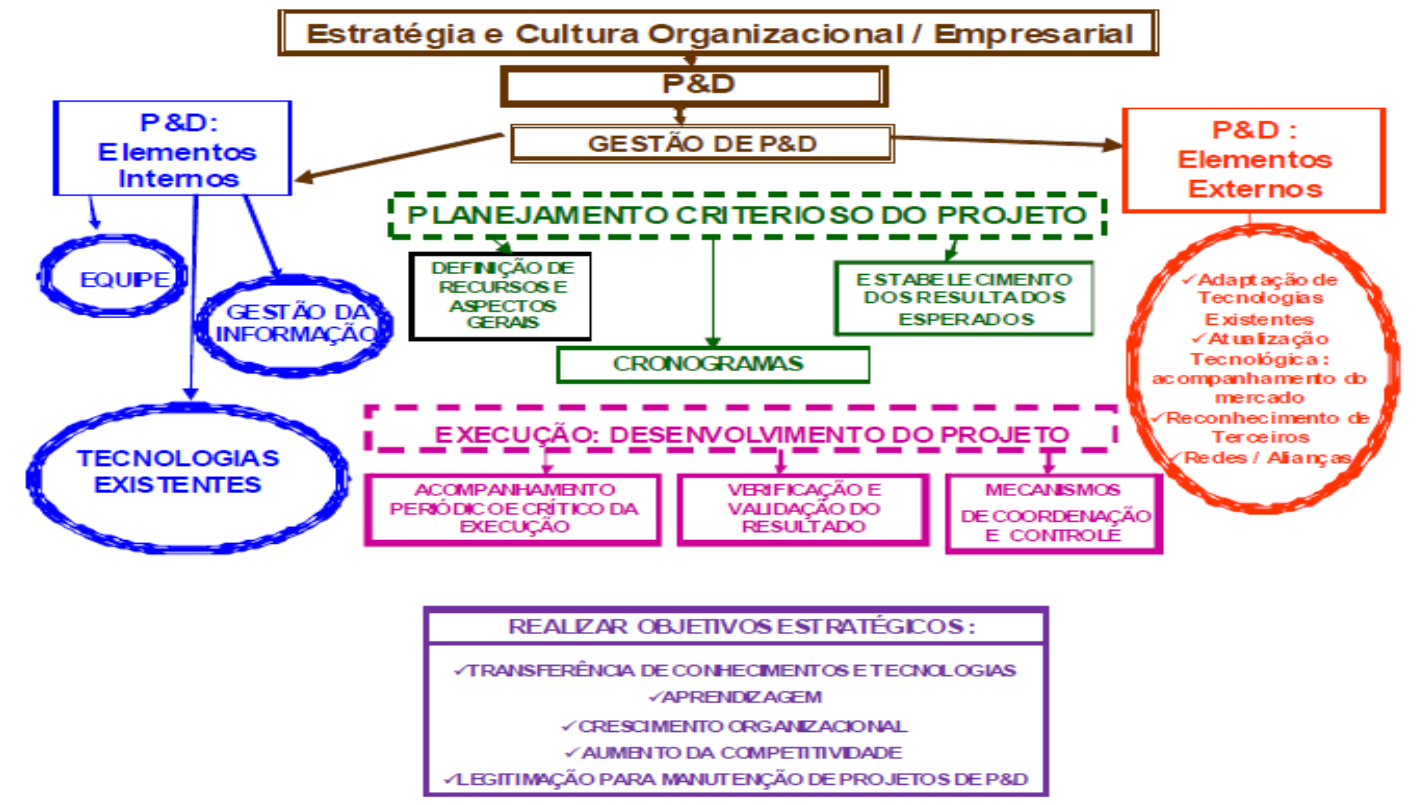

Figura 2: Modelo para análise dos dados

Fonte: DENDENA (2011)

Em síntese, a literatura relata que as organizações que investem e gerenciam as atividades de $\mathrm{P} \& \mathrm{D}$, inicialmente, realizam o planejamento do projeto, o que inclui, entre 
outros aspectos, a definição de recursos e do cronograma de execução e o estabelecimento dos resultados esperados. Durante o desenvolvimento do projeto, destacam-se três processos: acompanhamento periódico e crítico da execução, verificação e validação dos resultados e implantação de mecanismos de coordenação e controle (COHEN; LEVINTHAL, 1989; LIYANAGE et al., 1999; HASEGAWA; FURTADO, 2006; JUNG; RIBEIRO; CATEN, 2008)

Além disso, a gestão de $P \& D$ deve prever a qualificação da equipe e de seu coordenador e valorizar as parcerias com outras instituições, de forma a alcançar os objetivos estratégicos e aumentar a competitividade de maneira mais eficiente (DENDENA, 2011), uma vez que o aprendizado advindo das alianças e redes contribui para a realização dos projetos de $\mathrm{P} \& \mathrm{D}$ ao possibilitar a troca de conhecimentos entre organizações (FREITAS et al., 1996; KAYMAKÇALAN, 2000; PEREIRA, 2000; SIQUEIRA, 2000; BATTELE INSTITUTE, 2001; TERRA; GORDON, 2002)

De especial relevância é o acompanhamento do desenvolvimento tecnológico, principalmente no que diz respeito à adaptação das tecnologias existentes no mercado para a realização de novos produtos, processos e metodologias (HAYAMI; RUTTAN, 1998; GOVINDARAJAN; TRIMBLE, 2006).

Ou seja, o modelo proposto para a análise dos dados procurou contemplar grande parte dos elementos necessários para uma gestão de $\mathrm{P} \& \mathrm{D}$ de quinta geração. O processo para análise dos seis projetos de $\mathrm{P} \& \mathrm{D}$ estudados em profundidade seguiu a seguinte ordem: contato com os gerentes do projeto, agendamento de entrevista, transcrição das entrevistas gravadas. Para facilitar a análise, foi construído um quadro com as variáveis do modelo teórico.

O levantamento dos resultados dos projetos considerou: produtos gerados; patentes solicitadas e registradas; livros publicados; dissertações de mestrado e teses de doutorado defendidas; percentual de redução nos custos em função de tecnologias desenvolvidas; pesquisas transformadas em oportunidades de negócios; número de projetos desenvolvidos com universidades ou centros de pesquisa e em parceria com a iniciativa privada. 


\section{Análise dos resultados}

Com relação à primeira dimensão do modelo teórico (planejamento do projeto), onde se incluem a definição de recursos, o cronograma e resultados esperados, os projetos de P\&D da CEMIG estão em conformidade com o que é preconizado pela literatura a partir do modelo de segunda geração de gestão de P\&D. De acordo com o Manual do Programa de Pesquisa e Desenvolvimento do Setor de Energia Elétrica, a submissão de projetos de P\&D à avaliação da ANEEL deve estar enquadrada em formulário específico que exige o detalhamento dos objetivos, resultados esperados e recursos.

No que diz respeito à segunda dimensão de análise (execução e desenvolvimento do projeto), três aspectos são ressaltados no modelo. Quanto ao primeiro - acompanhamento periódico e crítico da execução do projeto - constata-se que, no caso dos projetos analisados, não há um padrão único. O acompanhamento realizado nos projetos estudados se deu por meio de reuniões, e-mails, telefonemas, conforme a percepção da necessidade pelo gerente do projeto, cabendo à ANEEL regulamentar o investimento no programa, acompanhar a execução dos projetos e avaliar seus resultados apenas no final dos mesmos.

O Quadro 2 resume os seis projetos de P\&D estudados. 


\begin{tabular}{|c|c|c|c|c|c|c|}
\hline & P\&D 003 & P\&D 006 & P\&D 023 & P\&D 051 & P\&D 084 & P\&D 110 \\
\hline TÍTULO & $\begin{array}{l}\text { Condutores } \\
\text { compactos: } \\
\text { otimização e } \\
\text { melhoria do } \\
\text { desempenho }\end{array}$ & $\begin{array}{l}\text { Aplicação e } \\
\text { disponibilizaçã } \\
\text { o dos dados de } \\
\text { monitora- } \\
\text { mento em } \\
\text { tempo real } \\
\text { de linhas de } \\
\text { transmissão }\end{array}$ & $\begin{array}{l}\text { Revitalização } \\
\text { de transforma- } \\
\text { dores de } \\
\text { Potência }\end{array}$ & $\begin{array}{l}\text { Usina } \\
\text { termelétrica } \\
\text { solar } \\
\text { experimental } \\
\text { de } \quad 10 \mathrm{KW} \\
\text { utilizando } \\
\text { concentradore } \\
\text { s cilíndricos } \\
\text { parabólicos }\end{array}$ & $\begin{array}{ll}\text { Predição e } & \\
\text { análise de } & \\
\text { informações } & \text { em } \\
\text { tempo real } & \text { de } \\
\text { linhas } & \text { de } \\
\text { transmissão } & \end{array}$ & $\begin{array}{l}\text { Pesquisa } \\
\text { aplicada em } \\
\text { tecnologia de } \\
\text { sensores } \\
\text { ópticos a fibra }\end{array}$ \\
\hline PARCEIROS & $\begin{array}{l}\text { Furukawa } \\
\text { Industrial } \\
\text { S.A. } \\
\text { FCO/UFMG }\end{array}$ & & ABB e UFMG & CEFET-MG & \begin{tabular}{l}
\multicolumn{2}{l}{ Universidade } \\
Federal de Juiz \\
de Fora
\end{tabular} & $\begin{array}{l}\text { CPQD - } \\
\text { Campinas }\end{array}$ \\
\hline OBJETIVOS & $\begin{array}{l}\text { Desenvolver } \\
\text { e } \\
\text { construir } \\
\text { protótipo de } \\
\text { cabo } \\
\text { condutor } \\
\text { compacto }\end{array}$ & $\begin{array}{l}\text { Desenvolver } \\
\text { metodologia de } \\
\text { monitora- } \\
\text { mento de linhas } \\
\text { de transmissão }\end{array}$ & $\begin{array}{l}\text { Desenvolver } \\
\text { metodologia } \\
\text { para } \\
\text { revitalização do } \\
\text { parque de } \\
\text { transforma- } \\
\text { dores de } \\
\text { potência }\end{array}$ & $\begin{array}{l}\text { Construir e } \\
\text { operar uma } \\
\text { mini-usina } \\
\text { termelétrica } \\
\text { solar }\end{array}$ & $\begin{array}{l}\text { Desenvolver } \\
\text { um programa } \\
\text { computacional } \\
\text { para } \\
\text { monitora- } \\
\text { mento das linhas } \\
\text { de tr. }\end{array}$ & $\begin{array}{l}\text { Protótipo: } \\
\text { sistema de } \\
\text { monitoração de } \\
\text { sensores a fibra } \\
\text { ótica para } \\
\text { supervisão de } \\
\text { tensão } \\
\text { mecânica }\end{array}$ \\
\hline $\begin{array}{l}\text { RESULTADOS } \\
\text { ESPERADOS }\end{array}$ & $\begin{array}{l}\text { Melhorar a } \\
\text { qualidade do } \\
\text { serviço; } \\
\text { recapacitar } \\
\text { LT; aumentar } \\
\text { a potência } \\
\text { transmitida } \\
\text { com menos } \\
\text { perdas } \\
\text { térmicas }\end{array}$ & $\begin{array}{l}\text { Aumentar a } \\
\text { capacidade de } \\
\text { transmissão de } \\
\text { LT existentes }\end{array}$ & $\begin{array}{l}\text { Conhecer o } \\
\text { Comportament } \\
\text { o dos } \\
\text { transformador. } \\
\text { em função do } \\
\text { envelhecimento } \\
\text { e propor } \\
\text { metodologia p/ } \\
\text { revitalizá-los }\end{array}$ & $\begin{array}{l}\text { Viabilizar uma } \\
\text { nova } \\
\text { fonte de } \\
\text { energia } \\
\text { elétrica } \\
\text { renovável e } \\
\text { diversificar o } \\
\text { parque } \\
\text { gerador }\end{array}$ & $\begin{array}{l}\text { Desenvolver } \\
\text { software } \\
\text { para } \\
\text { monitoramento } \\
\text { de linhas de } \\
\text { transmissão }\end{array}$ & $\begin{array}{l}\text { Localizar } \\
\text { falhas ao longo } \\
\text { da rede para } \\
\text { redução de } \\
\text { custos de } \\
\text { manutenção }\end{array}$ \\
\hline INÍCIO & 2000 & 2000 & 2001 & 2001 & 2003 & 2003 \\
\hline FIM & 2005 & 2002 & 2006 & 2007 & 2006 & 2006 \\
\hline PATENTE & Solicitada & Não & Não & Não & Solicitada & Solicitada \\
\hline RESULTADOS & $\begin{array}{l}\text { Construção } \\
\text { de } \\
\text { linha } \\
\text { experimental }\end{array}$ & $\begin{array}{l}\text { Domínio do uso } \\
\text { da tecnologia } \\
\text { importada p/ } \\
\text { monitora/ das } \\
\text { LT }\end{array}$ & $\begin{array}{l}\text { Desenvolvimen } \\
\text { to de um } \\
\text { transformador } \\
\text { refrigerado a } \\
\text { óleo } \\
\text { vegetal }\end{array}$ & $\begin{array}{l}\text { Diversificação } \\
\text { do parque } \\
\text { gerador } \\
\text { através da } \\
\text { nova fonte } \\
\text { de energia } \\
\end{array}$ & $\begin{array}{l}\text { Protótipo de } \\
\text { sistema } \\
\text { computacionais e } \\
\text { programa }\end{array}$ & $\begin{array}{l}\text { Protótipo } \\
\text { inédito de } \\
\text { monitoramento } \\
\text { que utiliza um } \\
\text { sensor ótico }\end{array}$ \\
\hline $\begin{array}{l}\text { RESULTADOS } \\
\text { ACADÊMICOS }\end{array}$ & & $\begin{array}{l}\text { Artigos } \\
\text { científicos } \\
\text { em congressos } \\
\text { nacionais }\end{array}$ & $\begin{array}{ll}\text { Artigos } & \\
\text { científicos } & \\
\text { em } & \\
\text { congressos } & \\
\text { nacionais } & \mathrm{e} \\
\text { tese } & \text { de } \\
\text { doutorado } & \end{array}$ & & $\begin{array}{l}\text { Artigos } \\
\text { científicos } \\
\text { em congressos } \\
\text { e dissertação de } \\
\text { mestrado }\end{array}$ & $\begin{array}{l}\text { Artigos } \\
\text { científicos } \\
\text { apresentados } \\
\text { em congressos }\end{array}$ \\
\hline
\end{tabular}

Quadro 2: Síntese dos projetos analisados

Fonte: Elaborado pelos autores 
Em outras palavras, a interação dos gerentes dos projetos de P\&D da CEMIG com os parceiros envolvidos ocorreu na medida do considerado necessário, com reuniões periódicas sem padrão definido e comunicação via e-mails e telefone, além do formulário da ANEEL enviado semestralmente à agência. Nos projetos analisados, não há registros formais das reuniões, seja por meio de atas ou relatórios, o que compromete a gestão da informação, o acompanhamento e a transferência dos progressos do projeto e das tecnologias ou metodologias desenvolvidas.

Quanto ao segundo aspecto - mecanismos de coordenação e controle - parece haver necessidade de ajustes quanto à formalização da responsabilidade e do papel de cada ator. Por exemplo, no projeto de $\mathrm{P} \& \mathrm{D} 003$ não houve contrato entre os parceiros e, sim, um acordo informal baseado na relação de confiança. Segundo o relato do gerente do projeto, esse foi um dos fatores que impediu alcançar-se o objetivo do projeto de P\&D.

Em relação aos imprevistos que podem surgir no desenvolvimento de qualquer projeto, não houve acordo de como lidar com eles. Ainda no projeto 003, a empresa parceira Furukawa foi vendida e a CEMIG não teve como continuar o projeto. De acordo com o depoimento do gerente do Projeto de P\&D 003: “A CEMIG, por exemplo, estava com uma viagem marcada para visitar a fábrica da NEXOS (empresa que adquiriu a Furukawa) na Bélgica, conversar sobre o cabo e mostrar os resultados da linha experimental. No entanto, quando os primeiros resultados dos protótipos da linha experimental saíram, houve uma mudança e a NEXOS não mais recebeu a CEMIG" (RELATO DO GERENTE DE P\&D 003, nov. 2009).

Quanto ao terceiro aspecto da segunda dimensão - verificação e validação dos resultados, os gerentes de $\mathrm{P} \& \mathrm{D}$ envolvidos nos seis projetos demonstraram preocupação em verificar os resultados em termos de capacitação das equipes e aquisição de conhecimento. No entanto, no que diz respeito aos impactos dos projetos nos parceiros industriais, não existe preocupação de verificação semelhante, apesar da importância potencial do programa de pesquisa CEMIG/ANEEL para a cadeia produtiva do setor elétrico brasileiro.

A terceira dimensão de análise engloba três aspectos: a equipe, a gestão da informação e a identificação das tecnologias já existentes. Com relação à equipe, foram analisados os seguintes aspectos: visão e perfil do gerente, capacitação e interação da equipe e comprometimento das áreas funcionais. 
Nos seis projetos analisados, os gerentes trabalharam praticamente sozinhos na CEMIG, com pouco envolvimento das áreas funcionais ao longo dos projetos. Esta é uma característica do modelo de gestão de $\mathrm{P} \& \mathrm{D}$ de primeira geração: uma gerência de $\mathrm{P} \& \mathrm{D}$ isolada, com grande autonomia. No entanto, os coordenadores dos projetos de P\&D mostraram-se motivados, mesmo que, ao coordenarem um projeto de $\mathrm{P} \& \mathrm{D}$, não recebam remuneração adicional e tenham suas atribuições aumentadas. O depoimento a seguir ilustra esta motivação: "Os projetos geram aprendizado para todos os envolvidos, e quem ganha com isso somos nós e a CEMIG (RELATO DO GERENTE DE P\&D 023, nov. 2009)". E ainda: "Eu gosto de lidar com tecnologia nova." (RELATO DO GERENTE DE P\&D 084, nov. 2009).

O segundo aspecto desta terceira dimensão de análise - a gestão da informação mostrou-se frágil nos projetos estudados. Constatou-se dificuldade de comunicação entre os setores da CEMIG envolvidos e seus parceiros, dificultando e atrasando os cronogramas e também a assimilação de conhecimentos e a troca de informações. Dos seis gerentes entrevistados, três acreditam que as informações e o conhecimento gerado ficaram restritos à área do projeto. Quanto à consideração das tecnologias existentes no gerenciamento dos projetos, ele se refere à transferência de tecnologias entre setores da organização e, dentre os seis projetos de $\mathrm{P} \& \mathrm{D}$ estudados, apenas um teve esta preocupação.

A quarta dimensão de análise se refere ao ambiente externo à organização, enfatizando as características referentes à adaptação de tecnologias existentes extramuros, à atualização tecnológica por meio do acompanhamento dos mercados, ao reconhecimento de terceiros e à formação de redes e alianças.

Com relação às tecnologias existentes externamente, seu acompanhamento é de grande importância para o desenvolvimento dos projetos, visto que se pode evitar duplicação de pesquisas e redução de custos. Dos seis projetos analisados, cinco adaptaram tecnologias existentes, acrescentando novos elementos às mesmas. Segundo o relato do gerente do projeto 096, em novembro de 2009, “(...) nós ficamos sempre a par, inclusive foram feitas viagens para a Noruega e para a França. Enquanto na CEMIG existe uma pessoa que lida com bombas de calor, na Noruega existem duzentos engenheiros que lidam exclusivamente com bombas de calor. Lá existe um intenso treinamento para que ocorra o desenvolvimento de tecnologias alternativas de energia".

Os gerentes de P\&D da CEMIG na maior parte das vezes têm recursos previstos nos projetos para visitar centros internacionais que apresentem avanço tecnológico ou 
excelência em P\&D. Há, inclusive, um gerente que, com recursos próprios, viajou para visitar empresas e centros de pesquisa, com o intuito de conhecer, avaliar e se atualizar tecnologicamente.

Finalmente, a dimensão Realizar Objetivos Estratégicos compreende cinco aspectos: transferência de conhecimentos e de tecnologias, aprendizagem, crescimento organizacional, aumento de competitividade e legitimação para manutenção de projetos de $P \& D$. Esses aspectos são os próprios objetivos do programa CEMIG/ANEEL. Observa-se a preocupação do setor elétrico com os benefícios organizacionais a serem trazidos pelos projetos, tanto na área ambiental, econômica, na ampliação de conhecimentos quanto na inovação. No entanto, ainda não há o amplo reconhecimento da estreita relação entre a atividade de $\mathrm{P} \& \mathrm{D}$ e os objetivos estratégicos da organização. 


\section{Conclusões}

A inovação é o objetivo último de qualquer programa empresarial de P\&D. Como dispõe o Manual dos Programas de P\&D do Setor Elétrico Brasileiro (ANEEL, 2001), “os programas de $P \& D$ devem estar pautados na busca de inovações para fazer frente aos desafios tecnológicos e de mercado das empresas de energia elétrica”. No entanto, no que diz respeito ao programa CEMIG/ANEEL, há necessidade de algumas modificações para que o sucesso em inovação seja alcançado.

De modo geral, os resultados de um projeto de $\mathrm{P} \& \mathrm{D}$ incluem capacitação de recursos humanos, criação ou aprimoramento de infra-estrutura de $\mathrm{P} \& \mathrm{D}$, geração de novos conhecimentos e desenvolvimento de novas tecnologias. Para as empresas de energia elétrica, esses resultados podem se converter em novos negócios e receitas, ganhos de produtividade, otimização de processos, melhoria da qualidade dos serviços prestados e redução de custos. Porém, observou-se nos projetos de P\&D estudados que os resultados gerados não foram, na maior parte das vezes, transferidos e colocados em prática.

No que diz respeito ao modelo de gerenciamento de $\mathrm{P} \& \mathrm{D}$ adotado pelo programa CEMIG/ANEEL, não foram reconhecidas características de uma determinada geração de modelo de gestão de $\mathrm{P} \& \mathrm{D}$, mas sim aspectos das diferentes gerações, apesar do modelo privilegiar características da segunda geração: i) Da primeira geração, a característica mais marcante constatada foi a autonomia dos gerentes na condução dos projetos, a partir de seus interesses específicos e de sua especialidade; ii) Da segunda geração de modelos de gestão de $\mathrm{P} \& \mathrm{D}$, percebeu-se a preocupação em ter-se projetos com objetivos bem definidos e períodos estabelecidos para sua realização; iii) Das características de gestão descritas como terceira geração de gerenciamento de $\mathrm{P} \& \mathrm{D}$, a que se sobressai na literatura é a integração da estratégia tecnológica e de $P \& D$ com a reflexão estratégica global da organização. No entanto, esta integração não é clara no que diz respeito aos projetos do programa CEMIG/ANEEL estudados; iv) A partir da denominada quarta geração de gestão de P\&D, o estabelecimento de parcerias para o desenvolvimento dos projetos é enfatizado.

Nos projetos analisados, em apenas um isto não ocorre. No entanto, não foram percebidas características como integração funcional e transferência interna de conhecimentos, que são indicadas pela literatura como conseqüência do fortalecimento das redes de relacionamento; e, v) Das características da quinta geração de gestão de $\mathrm{P} \& \mathrm{D}$, não foi identificada a preocupação com a integração estratégica e a adaptação dos projetos à velocidade das mudanças na indústria. 
Em resumo e tomando como referência o modelo conceitual de análise dos dados adotado, a Figura 3 apresenta o processo de gestão de P\&D da CEMIG nos casos analisados.

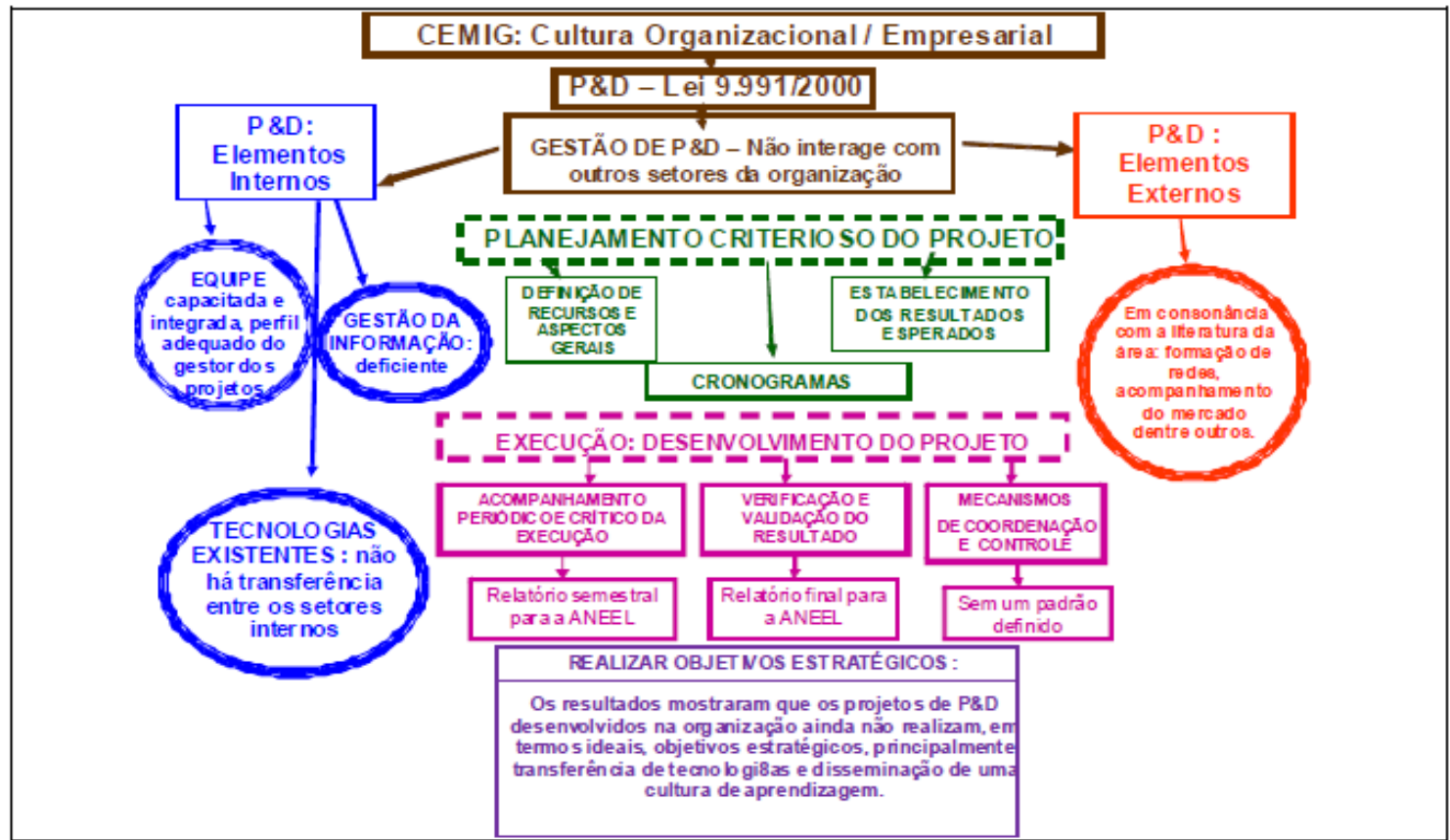

Figura 3: Modelo adotado pela CEMIG

Fonte: Elaborado pelos autores.

A motivação dos gerentes dos projetos de $\mathrm{P} \& \mathrm{D}$ analisados é evidente. Por outro lado, os parceiros dos projetos, na maioria dos casos, já tinham relacionamentos anteriores com esses gerentes, o que certamente facilita o gerenciamento. Já quanto à proteção da propriedade industrial, os gerentes mostraram pouca preocupação com a solicitação de patentes, pois acreditavam não ser uma prioridade. O gerenciamento dos projetos é prejudicado pelo acúmulo de funções dos gerentes e a pouca inter-relação entre os setores e áreas funcionais da organização.

As equipes dos projetos não seguem um padrão único na periodicidade e na forma de troca de informações. Se por um lado, a flexibilidade na condução dos projetos apresenta aspectos positivos, por outro pode prejudicar a disseminação das informações e a transferência de conhecimento.

Com relação ao acompanhamento periódico e crítico do projeto, mesmo considerando que relatórios devam ser enviados semestralmente à ANEEL, este não ocorre no decorrer do projeto. A obrigatoriedade da Lei $n^{\circ}$ 9.991, de 24 de julho de 2000 
(BRASIL, 2000) foi apontada por todos os gerentes entrevistados como sendo ainda a razão maior da existência dos projetos de $P \& D$ e, por fim, a exigência de prestação de contas meticulosa sobre toda contratação de serviços pela CEMIG gera morosidade e dificulta o desenvolvimento de redes de parcerias. 


\section{Referências}

AENOR (ASOCIACIÓN ESPAÑOLA DE NORMALIZACIÓN Y CERTIFICACIÓN). UNE 166002 EX: Gestión de la I+D+I: Requisitos del Sistema de Gestión de la I+D+I. Madrid: UNE, 2002.

ARNOLD, E. GUY, K. Parfallel Convergence: National Strategies in Information Technology. London: Frances Pinter, 1986.

BATTELE INSTITUT. Prospecção tecnológica: Melhores negócios do futuro, desafios e oportunidades. MCT - Parcerias Estratégicas, Brasília, Centro de Gestão e Estudos Estratégicos do Ministério da Ciência e Tecnologia, n. 11, p. 136-150, junho 2001. Organizador: CGEE:Centro de Gestão e Estudos Tecnológicos.

BRASIL. Lei $n^{\circ}$ 9.991, de 24 de julho de 2000. Dispõe sobre realização de investimentos em pesquisa e desenvolvimento e em eficiência energética por parte das empresas concessionárias, permissionárias e autorizadas do setor de energia elétrica, e dá outras providências. Diário Oficial da União, 25 jul. 2000. Disponível em: <http://www.planalto.gov.br/ccivil_03/Leis/L9991.htm>. Acesso em: 13 mar. 2010.

BURGESS, J.; SMITHAM, J. BHP’s R\&D Management philosophy. R\&D Review, v. 2, p. 11-12, set. 1995.

BURNS, T.; STALKER, C. M. The Management of Innovation. London: Tavistock Publications, 1961.

CARVALHO, A. M. Definição de Temas Tecnológicos para Pesquisa e Desenvolvimento: caso CEMIG. 2008. Dissertação (Mestrado em Administração de Empresas) - Faculdade de Administração de Empresas, Pontifícia Universidade Católica de Minas Gerais, Belo Horizonte, 2008.

CONTRACTOR, F. J.; LORANGE, P. Cooperative Strategies in International Business. Lexington, MA: Lexington Books, 1988.

COOPER, R. G.; EDGET, S. J.; KLEINSCHMIDT, E. J. Winning business in product development: the critical success factors. Research - Technology Management, v. 39, n. 4, p.18-29, 1996.

COOPER, R. G.; EDGET, S. J.; KLEINSCHMIDT, E. J. Portfolio Management in newproduct development: lessons from the leaders, 2. Research Technology Management, Oregon, v. 40, n. 6, p. 43-52, 1997.

COOPER, R. G.; EDGET, S. J.; KLEINSCHMIDT, E. J. New Product Portfolio Management: Practices and Performance. Journal Product Innovation Management, n. 16, p. 333-351, 1999.

COUTINHO, Paulo Luiz de Andrade. Estratégia Tecnológica e Gestão da Inovação: uma estrutura analítica voltada para os Administradores de Empresas, 2004. Tese (Doutorado 
em Engenharia Química) - Faculdade de Química, Universidade Federal do Rio de Janeiro, Rio de Janeiro, 2004.

CRAWFORD, C. M. The Hidden Costs of Accelerated Product Development. Journal of Product Innovation Management, v. 9, p. 188-99, 1992.

DANNEELS, E.; KLEINSCHMIDT, E. J. Product innovativeness from the firm's perspective. Journal of Product Innovation Management, v. 8, n. 6, p. 357-374, 2001.

DENDENA, Adriana Carvalho de Menezes. Gestão de projetos de P\&D: proposta de um modelo. Revista Interação, v. 13, n. 13, p. 13-29, 2011.

DODGSON, M. Technological Collaboration in Industry. London: Rotledge, 1993.

DRUCKER, P. The discipline of innovation. Harvard Business Review, v. 76, n. 4, p. 73 84, 1998.

DUMAINE, B. How managers can succeed through speed. Fortune, n. 13, Feb. 1989.

FREITAS FILHO, A. F. et al. Parceria como Modelo de Cooperação entre Instituições de P\&D. In: SIMPÓSIO DE GESTÃO DA INOVAÇÃO TECNOLÓGICA, 1996, São Paulo. Anais... São Paulo, nov. 1996.

GODOY, A. S. A Pesquisa Qualitativa e sua Utilização em Administração de Empresas. Revista de Administração de Empresas, São Paulo, v. 35, n. 4, 1995.

GOVINDARAJAN, V.; TRIMBLE, C. Os 10 Mandamentos da Inovação Estratégica - do conceito à implementação. Rio de Janeiro: Campus, 2006.

HASEGAWA, Mirian; FURTADO, André Tosi. Avaliação dos Impactos de Programas de P\&D. Inovação Uniemp, Campinas, v. 2, n. 3, jul./ago. 2006.

HASKLISCH, C. S.; FUSFELD, H. I.; LEVINSON, A. D. Trends in Collective Industrial Research. Centre for Science and Technology Policy, Graduate School of Business Administration. New York: New York University, 1986.

HAYAMI, Y.; RUTTAN, V. W. Desenvolvimento agrícola: teoria e experiências internacionais. Tradução Maria Vittoria von Bulow e Joachim S. W. Von Bulow. Brasília: Embrapa, 1988.

IRVINE, J.; MARTIN, B. R. Foresight in Science, Picking the Winners. London: Printer, 1984.

JANNUZZI, G. M. Uma avaliação das atividades recentes de P\&D em energia renovável no Brasil e Reflexões para o Futuro. Energy Discussion Paper, n. 2, p. 64, jul. 2003.

JUNG, C. F.; RIBEIRO, J. L. D.; CATEN, C. S. Análise de um Modelo para Pesquisa e Desenvolvimento de Inovações Tecnológicas voltado ao Desenvolvimento Regional. 2008. Disponível em: <www.abepro.org.br/enegep2008>. Acesso em: 22 ago. 2010. 
KAYMAKÇALAN, Ömer. Knowledge Management in Research and Technology Organizations: Policies and practices to make your RTO competitive in the $21^{\text {st }}$ Century. In: Group Meeting on Initiatives for $S$ and T Capacity Building for the 21st Century. Beirute, Líbano, nov. 2000, v.2, p.117-126. Disponível em: <http://www.waitro.org/Publications/Others/kaymac.htm>. Acesso em: 22 ago. 2010.

LICHTENTHALER, E. Third generation management of technology intelligence processes. $R \& D$ Management, v. 33, n. 4, 2003.

LIMA, Isaura Alberton. Gestão de Projetos de Pesquisa e Desenvolvimento no Âmbito da Cooperação Escola-Empresa. Dissertação (Mestrado em Tecnologia) - Programa de PósGraduação em Tecnologia, Centro Federal de Educação Tecnológica do Paraná, Unidade de Ponta Grossa, Curitiba-PR, 1999.

LIYANAGE, S; GREENFIELD, P.; DON, R. Towards a fourth Generation R\&D Management model-research networks in Knowledge Management. Journal of Technology Management, v. 18, n. 3, 1999.

METCALF, J. S.; BODEN, M. Impulse and Diffusion in the Study of Technological Change. The Economics of Innovation, v. 50, p. 707-717, 1991.

MOWERY, D.; ROSENBERG, N. The influence of market demand upon innovation: a critical review of some recent empirical studies. Research Policy, v. 8, p. 102-153, 1979.

NELLORE, R. BALACHANDRA, R. Factors influencing success in integrated product development (IPD) projects. IEEE Transactions on Engineering Management, v.48, n. 2, p. 164-173, 2001.

PEREIRA, H. M. S. Parceria Tecnológica sob o Olhar da Propriedade Intelectual: Objetivo, Objeto e Seleção de Parceiros. In: SIMPÓSIO DE GESTÃO DA INOVAÇÃO TECNOLÓGICA, 21., 2000, São Paulo. Anais... São Paulo, 2000.

REED, R.; LEMARK, D. L. MONTGOMERY, J. C. Beyond process: TQM content and firm performance. Academy of Management Review, v. 21, n. 1, p. 173-202, 1996.

REINER, G. Winning the Race for New Product Development. Management Review, v. 78 , n. 8, p. 52-3, 1989.

REIS, D. R. Gestão da Inovação Tecnológica. São Paulo: Manole, 2008.

RENAULT, T. et al. Gestão da Inovação - um esforço de P\&D em Empresa Distribuidora de Energia Elétrica. ENGEVISTA, v. 9, n. 2, p. 100-111, dez. 2007.

RICHARDSON, R. J. Pesquisa Social: métodos e técnicas. São Paulo: Atlas, 1999.

ROOME, N. Business strategy, R\&D management and environmental imperatives. $R \& D$ Management, v. 24, p. 65-81, 1994. 
ROTHWELL, R. External Networking and Innovation in Small and Medium-sized Manufacturing Firms in Europe. Technovation, v. 11, n. 2, p. 93-112, 1991.

ROTHWELL, R. Towards the fifth-generation innovation process. International Marketing Review, v. 11, n. 1, p. 7-31, 1994.

ROUSSEL, P. A.; SAAD, K. N.; BOHLIN, N. Third Generation R\&D. Boston: MA, Arthur D. Little/Harvard Business School Press, 1991.

ROUSSEL, P. A.; SAAD, K. N.; BOHLIN, N. Pesquisa e Desenvolvimento: como integrar P\&D ao plano estratégico e operacional das empresas como fator de produtividade e competitividade. São Paulo: Makron Books, 1992.

RUDOLPH, S. E. What Smart Companies are Doing in New Product Development. Cambridge, MA: Centre for Product Development/Arthur D. Little, 1989.

SALTER, A. J.; MARTIN, B. R. The economic benefits of publicly funded basic research: a critical review. Research Policy, Brighton, v. 30, n. 3, p. 509-532, 2001.

SAWHNEY, M.; PRANDELLI, E. Communities of creation: managing distributed innovation in turbulent markets. California Management Review, v. 42, n. 4, p. 45-69, 2000 .

SETHI, R.; NICHOLSON, C. Structural and contextual correlates of charged behavior in product development teams. Journal of Product Innovation Management, v. 18, n. 3, p. 154-168, 2001.

SIQUEIRA, P. C. As empresas de pesquisa sob contrato: um exemplo de integração pesquisa / indústria. Parcerias Estratégicas, n. 8, p. 55-83, maio 2000.

TERRA, J. C. C.; GORDON, C. Portais Corporativos: A Revolução na Gestão do Conhecimento. São Paulo: Negócio, 2002. 